\title{
Update on neuroimaging in disorders of consciousness
}

\author{
Leandro R.D. Sanz ${ }^{\mathrm{a}, \mathrm{b}}$, Aurore Thibaut ${ }^{\mathrm{a}, \mathrm{b}}$, Brian L. Edlow ${ }^{\mathrm{c}, \mathrm{d}}$, \\ Steven Laureys ${ }^{\mathrm{a}, \mathrm{b}, *}$, and Olivia Gosseries ${ }^{\mathrm{a}, \mathrm{b}, *}$
}

\begin{abstract}
Purpose of review
Neuroimaging has acquired a prominent place in the assessment of disorders of consciousness (DoC). Rapidly evolving technologies combined with state-of-the-art data analyses open new horizons to probe brain activity, but selecting appropriate imaging modalities from the plethora of available techniques can be challenging for clinicians. This update reviews selected advances in neuroimaging that demonstrate clinical relevance and translational potential in the assessment of severely brain-injured patients with DoC.

\section{Recent findings}

Magnetic resonance imaging and high-density electroencephalography provide measurements of brain connectivity between functional networks, assessments of language function, detection of covert consciousness, and prognostic markers of recovery. Positron emission tomography can identify patients with preserved brain metabolism despite clinical unresponsiveness and can measure glucose consumption rates in targeted brain regions. Transcranial magnetic stimulation and near-infrared spectroscopy are noninvasive and practical tools with promising clinical applications.
\end{abstract}

\section{Summary}

Each neuroimaging technique conveys advantages and piffalls to assess consciousness. We recommend a multimodal approach in which complementary techniques provide diagnostic and prognostic information about brain function. Patients demonstrating neuroimaging evidence of covert consciousness may benefit from early adapted rehabilitation. Translating methodological advances to clinical care will require the implementation of recently published international guidelines and the integration of neuroimaging techniques into patient-centered decision-making algorithms.

\section{Keywords}

coma, diagnosis, disorders of consciousness, neuroimaging, prognosis

\section{INTRODUCTION}

Disorders of consciousness (DoC) are a group of rare conditions that encompass a wide range of severe acquired brain injuries. DoC affect a heterogeneous clinical population with varying etiologies, degrees of chronicity, and associated neurological deficits, which poses a challenge to the development of diagnostic, prognostic, and therapeutic guidelines $\left[1,2^{-"}, 3^{*}\right]$. The lack of rehabilitation infrastructures and trained healthcare professionals as well as the intricate ethico-legal issues in their management further contribute to the uncertain status of these vulnerable patients, who are too often neglected by healthcare systems $[4,5]$.

An accurate diagnosis is the first step toward personalized and optimized care. Diagnostic categories have been established based on clinical characteristics displayed by patients, although the consensual nomenclature and the list of behaviors indicating consciousness are continuously evolving in the light of new evidence $\left[6^{*}, 7^{*}, 8^{*}, 9\right]$. The unresponsive wakefulness syndrome (UWS), also known as the vegetative state, describes patients with

\footnotetext{
${ }^{a}$ Coma Science Group, GIGA Consciousness, University of Liège, ${ }^{\mathrm{b}}$ Centre du Cerveau ${ }^{2}$, University Hospital of Liège, Liège, Belgium, ${ }^{\mathrm{c}}$ Center for Neurotechnology and Neurorecovery, Department of Neurology, Massachusetts General Hospital and Harvard Medical School, Boston and ${ }^{d}$ Athinoula A. Martinos Center for Biomedical Imaging, Massachusetts General Hospital and Harvard Medical School, Charlestown, Massachusetts, USA

Correspondence to Dr Olivia Gosseries, Coma Science Group, GIGA B34, CHU de Liège, 1, avenue de l'Hôpital, 4000 Liège, Belgium.

E-mail: ogosseries@uliege.be

*Steven Laureys and Olivia Gosseries contributed equally.

Curr Opin Neurol 2021, 34:488-496
}

DOI:10.1097/WCO.0000000000000951 


\section{KEY POINTS}

- Neuroimaging is essential in the diagnostic and prognostic assessment of patients with disorders of consciousness.

- Multimodal imaging protocols providing complementary data are recommended by current guidelines to reduce misdiagnosis rates and leverage the strengths of each technique.

- Brain imaging and electrophysiology can monitor a treatment's efficacy and provide surrogate biomarkers of early responsiveness.

- Clinically unresponsive patients with a capacity for covert consciousness can be detected with neuroimaging and may benefit from adapted care plans to promote their recovery.

- Future evidence-based decision-making algorithms should guide the clinician in the use of neuroimaging techniques for brain-injured patients, both at the acute and the chronic stages.

preserved arousal (i.e., eye opening) but absent reproducible signs of consciousness [10-12]. The reappearance of unambiguous but low-level signs of consciousness marks the transition to the minimally conscious state minus (MCS-) [13], whereas the presence of residual language-related behaviors defines the MCS plus (MCS+) [14,15]. Emergence from MCS, and therefore from DoC is attained when a patient regains the ability to functionally communicate or use objects [16]. A substantial percentage of those patients experience ongoing cognitive deficits, and diagnostic criteria for the confusional state after traumatic brain injury were defined recently [17]. The recommended clinical scale to assess these behaviors is the Coma Recovery Scale Revised [18], which substantially reduces misdiagnosis rates compared to clinical consensus [19]. Complementary behavioral tools have been developed to overcome some of its pitfalls (e.g., its long administration duration) or provide additional information at the bedside (e.g., on pain perception or early motor behaviors) [20-25].

As our understanding of the neural networks underlying consciousness progresses, a growing body of evidence suggests that neurophysiological and neuroimaging measures should be taken into account in the diagnosis of DoC, as recommended in the recent guidelines of the European Academy of Neurology (EAN) and the American Academy of Neurology [1,2"']. Indeed, it has been demonstrated that a substantial fraction of clinically unresponsive patients displays brain activity suggesting the presence of covert consciousness, which has led to the proposal of new diagnostic taxons (e.g., 'cognitive motor dissociation' [26], 'covert cortical processing' [27], 'MCS*' [28]).

Brain imaging paradigms probing consciousness can be divided into 3 categories:

(1) Active paradigms record willful modulation of brain activity by the subject in response to a command, and in rare cases can be used to achieve communication via brain-computer interfaces [29]. These experiments tend to have a high specificity but a low sensitivity to detect conscious awareness, given the many confounding factors, such as aphasia and fluctuating vigilance.

(2) Passive paradigms measure brain responses to external stimuli without the participation of the subject. Although they assess the ability of a primary sensory brain region to process information, these paradigms do not demonstrate the presence of consciousness, which relies on higher-order associative cortical networks [30].

(3) Resting-state measurements study brain activity in the absence of tasks or stimulations. As they do not depend on active participation of the patients or their ability to perceive external stimulations, they mitigate the influence of common confounding factors, such as hearing or vision impairments. However, emerging insights into the interplay between brain networks at rest are only starting to allow the translation of these paradigms into clinically actionable data [31].

The study of activity and connectivity patterns across specific brain regions has reframed the historical search for 'consciousness hotspots' [32,33], which must now account for the influence of interconnected functional networks essential to the generation and maintenance of consciousness (e.g., the default mode, frontoparietal, ventral and dorsal attention networks, as well as corticothalamic feedback loops and the anterior forebrain mesocircuit).

Neuroimaging or neurophysiological exams are not limited to diagnostic uses, as they provide prognostic indicators of recovery $\left[34^{\prime}, 35,36^{\mathbf{\prime \prime}}, 37^{\mathbf{*}}, 38\right]$, surrogate markers of therapeutic efficacy $\left[39^{\prime \prime}, 40,41^{\prime \prime}, 42\right]$ and neural benchmarks of clinical assessments $\left[7^{\mathbf{*}}, 8^{\mathbf{*}}, 43\right]$. This review highlights a selection of recent original and review articles identified with focused searches of the literature in MEDLINE, associated references and personal libraries. We review common and emerging neuroimaging techniques that are paving the way for future multimodal frameworks and improved care for patients with DoC. 


\section{ELECTROPHYSIOLOGY}

Electroencephalography (EEG) is an accessible, portable, and noninvasive tool to probe cortical activity with a high temporal resolution. High-density montages in combination with artificial intelligence, source localization or functional connectivity analyses have opened new avenues in EEG signal processing [44]. The plethora of EEG markers available to date allows the development of multivariate classification techniques driven by machine learning algorithms, which may complement and sharpen the clinician's judgment $\left[45^{\boldsymbol{\prime}}, 46\right]$.

Nevertheless, well-established methods such as conventional clinical EEG or event-related potentials (ERPs) remain essential in the initial assessment of unresponsive patients when recorded and interpreted correctly, as highlighted in a review endorsed by the International Federation of Clinical Neurophysiology [3"']. Some ERP components perform better than others to detect consciousness, and the 2020 EAN guidelines consider the P300 more contributive than the mismatch negativity to differentiate between UWS and MCS patients, whereas they underline the relatively low sensitivity of all ERPs [2"-]. However, recent evidence indicates that using multisensory ERPs as well as single-subject personalized thresholds improves the accuracy of P300 assessments in chronic DoC patients [47"]. Moreover, ERPs can be combined with dynamic network connectivity analyses to categorize DoC patients, which has revealed impaired top-down processing and frontoparietal connectivity among chronic UWS compared to MCS patients (in line with previous results [48]), associated with altered middle-to-late ERP components linked to higher-order emotional processing (P3a and late positive potential) [49]. This combined approach illustrates the recent shift in experimental design from single-component ERP recordings to multidimensional assessments of cognitive and speech processes, as recently highlighted in a comprehensive review [50"].

By comparing activity patterns elicited by a passive movie-watching task, EEG may provide a practical and naturalistic bedside tool to detect covert cortical processing in chronic unresponsive patients [51"]. Most previous EEG-based protocols to identify cognitive motor dissociation have used active tasks requiring willful modulation of brain activity, and this novel individualized paradigm might be less affected by attentional confounding factors [36"',52-54]. Similarly, EEG brain activation to spoken commands can be identified in the acute phase using machine learning and predict functional outcomes at 12 months [36"']. These results highlight the prime importance of language function in the recovery of DoC patients, but common confounding factors such as endotracheal tubes or aphasia represent major hurdles to assess speech in this population. To address this issue, a variety of EEG methods have been developed to record the cortical tracking of speech $\left[37^{\bullet}, 55,56\right]$, and support vector machine automated classifiers may provide an unbiased alternative to human-based assessments [57"].

Additionally, EEG can be used to assess the integrity of sleep-wake cycles over 24 -h recordings and identify early polysomnographic signs of recovery [58]. The presence of sleep spindles has been previously associated with neuroimaging evidence of covert consciousness [59], but further advances in sleep data analysis will be necessary to translate this technique to the clinic, as the sleep architecture of DoC patients differs significantly from normal sleepwake patterns and shows an important heterogeneity [60"].

Combined with transcranial magnetic stimulation, EEG can be used to characterize the brain's neural response to magnetic perturbation [61]. This diagnostic measure, which reliably distinguishes between different levels of consciousness and detects covert consciousness [62"], was found to correlate with subcortical atrophy [63]. It also provides a singular opportunity to study mechanistic changes (e.g., decreased evoked slow-activity) induced by concomitant treatment such as transcranial electrical stimulation [39"].

EEG measures such as alpha power, variability, or reactivity have demonstrated prognostic value in the short-to-medium term, but data on long-term recovery are lacking $\left[38,64^{\prime \prime}\right]$. Initial insights indicate that high-density EEG functional connectivity assessments based on graph theory in prolonged DoC could outperform clinical predictions on 2-year outcomes [34"]. Future diagnostic and prognostic methods may increase their accuracy by integrating complementary spectral, complexity, and connectivity measures into multivariate machine learning models [46].

In summary, a combination of novel personalized paradigms, multisensory stimulations and stateof-the-art data analysis methods are setting the stage for future clinical decision-making pipelines based on sequential complementary neurophysiological assessments [3"'] (e.g., as proposed in Fig. 1).

\section{POSITRON EMISSION TOMOGRAPHY}

Resting-state fluorodeoxyglucose positron emission tomography (FDG-PET) plays an important role in the multimodal assessment of patients with DoC, as it provides a minimally invasive measure of glucose uptake across the whole brain. It also conveys the 


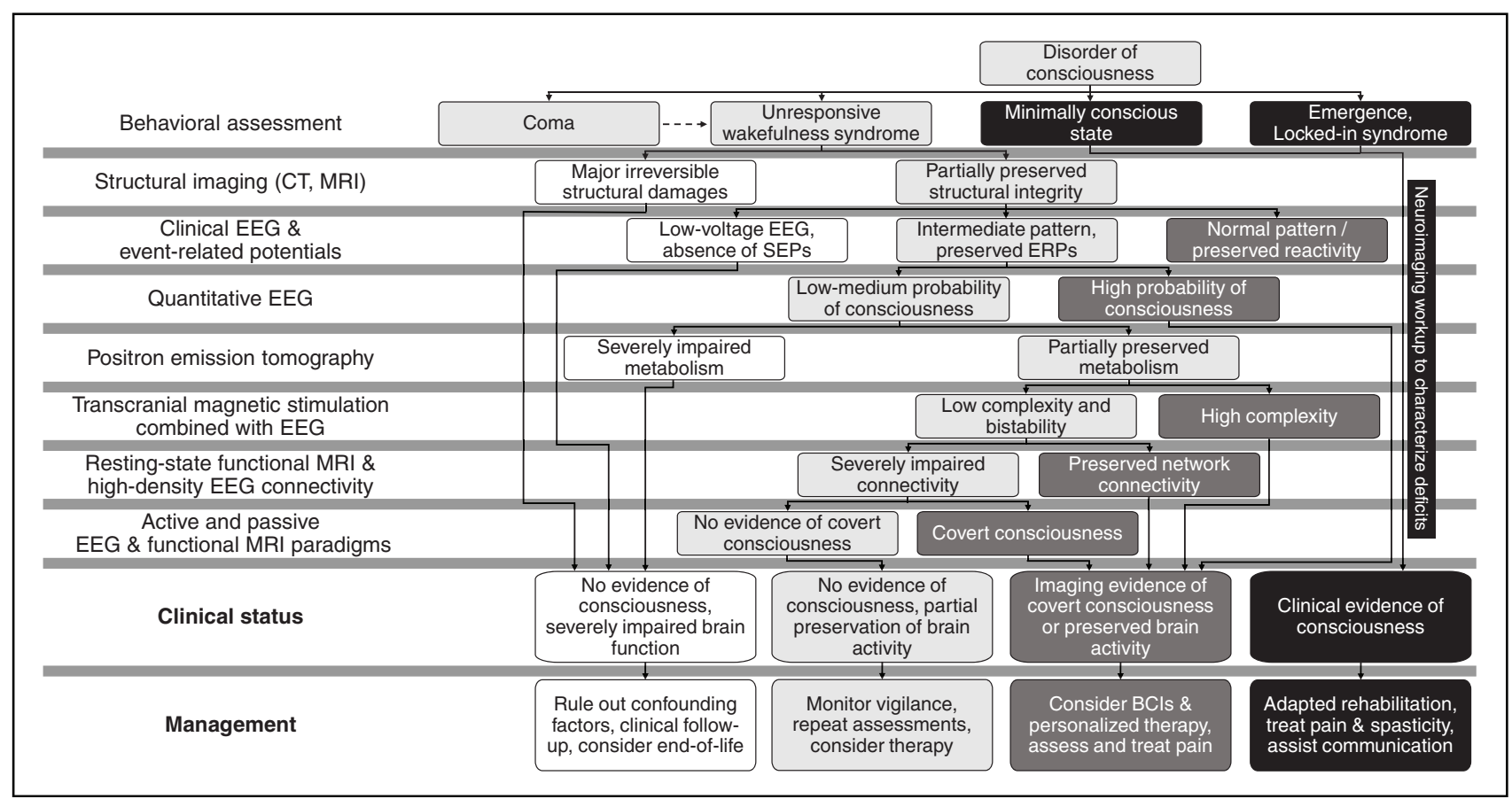

FIGURE 1. Illustrative multimodal decision tree guiding diagnostic exams for patients with disorders of consciousness. A sequential approach is proposed to optimize the allocation of neuroimaging resources, improve the detection rate of covert consciousness and guide the selection of therapies. White boxes indicate patients with severely impaired brain activity and poor prognosis; Light grey boxes indicate patients with uncertain capacity for consciousness, or mismatching neuroimaging results (e.g., unresponsive patient with preserved brain metabolism but impaired connectivity). Dark grey boxes indicate patients with neuroimaging-based evidence of consciousness or preserved brain activity compatible with conscious processing. Black boxes indicate patients with clinical signs of consciousness. CT, computerized tomography; MRI, magnetic resonance imaging; EEG, electroencephalography; SEPs, somatosensory evoked potentials; ERPs, event-related potentials; BCls, braincomputer interfaces. Inspired by [100] and [3"'].

advantage of reporting brain activity that occurred shortly before the scan, which minimizes the influence of sedation on the interpretability of the data [65].

The recent EAN guidelines underline the high sensitivity and high specificity of FDG-PET to differentiate UWS from MCS patients, when optimal technical conditions are met [2"']. Emerging evidence also shows that a significant proportion of unresponsive patients demonstrate resting-state cerebral metabolic rates compatible with consciousness, a condition termed 'nonbehavioral MCS' or 'MCS'' $[66,67]$. Similar to the terms 'cognitive motor dissociation' [26] and 'covert cortical processing' [27] (clinically UWS or MCS- patients with appropriate brain response to active and passive paradigms, respectively), this term marks the emergence of a new nomenclature based on neuroimaging criteria, which reflects the growing importance of paraclinical assessments in the appraisal of consciousness [6"]. These considerations call into question the goldstandard status of behavioral diagnosis, as covert cognitive processing missed by standardized clinical scales might be more frequent than previously thought [68]. For instance, the heartbeat-evoked response, an EEG measure of perceptual and selfconsciousness, was found to correlate better with FDG-PET-based diagnosis than behavioral diagnosis, which could indicate an ability to probe covert consciousness better than overt consciousness [69].

Although whole-brain metabolism provides a global measure of glucose consumption, FDG-PET data also offer the possibility to perform regional analyses and study local metabolism in key areas for a particular function [70]. Using this approach, a study recently showed higher metabolic rates among MCS + compared to MCS - patients in the left middle temporal cortex, a key region for semantic language processing [71"].

Beyond its utility to diagnose levels of consciousness, FDG-PET can be used to provide a neural benchmark for clinical observations. UWS patients confirmed by brain hypometabolism systematically obtained low scores on the Nociception Coma Scale 
- Revised (a validated clinical scale to assess pain perception in DoC patients), whereas their regional metabolism in the left insula was lower than for patients who displayed a potential capacity to perceive pain [43].

Despite its limited accessibility and its irradiating nature, FDG-PET has become a cornerstone in the assessment of consciousness. By detecting patients with relative preservation of brain metabolism in the early stage, FDG-PET could reduce misdiagnosis rates and lower the risk of premature withdrawal of life-sustaining therapy. In addition, FDG-PET can be used as a pharmacodynamic biomarker of targeted therapeutic interventions [72], thereby facilitating the development of new therapies aimed at promoting recovery of consciousness.

\section{MAGNETIC RESONANCE IMAGING}

Structural magnetic resonance imaging (MRI) offers a three-dimensional high-resolution characterization of the grey and white matter alterations following acquired brain injury, whereas functional MRI (fMRI) investigates neural activity at rest or in response to passive and active paradigms. Recent analytic methods have opened new avenues in the characterization of injured brain tissue (e.g., lesion mapping, morphometry, diffusion tensor imaging) and impaired functional connectivity across brain networks [73"- . These methods have evolved from static approaches to dynamic connectivity analyses that measure temporally varying patterns of brain activity at rest $[74,75]$.

The increase in spatial resolution provided by new-generation $7 \mathrm{~T}$ scanners will allow the future development of new microstructural analyses, highresolution connectometry and graph-theoretic network topology studies to track disease progression and recovery, as shown in a recent case report [76]. Meanwhile, diffusion tensor imaging has already shown potential as a prognostic marker for unconscious patients after a cardiac arrest, accurately predicting clinical outcomes at 6 months [35]. This technique has also revealed disruption of white matter connections within subcortical and cortical networks in acute and chronic DoC patients, correlating with clinical severity $\left[77,78^{\bullet}, 79,80\right]$.

The increasing number of publications reporting resting-state fMRI measures in DoC patients reflects the prolific contribution of this modality to our understanding of the brain properties that are necessary to sustain consciousness. Using cutting-edge functional connectivity analyses, recent studies on DoC patients have measured a number of changes in time-delay latency [81], large-scale network topological properties [82], fractal dimension of networks
[83], and variance of network metrics and dynamics $[75,84]$, compared to healthy controls. Decreased dynamic functional connectivity has been observed in the sensory and somatomotor networks, and abnormal network properties correlate with behavioral scores $[85,86]$, which demonstrates the potential clinical relevance of these surrogate biomarkers. Additionally, networks displayed reduced functional diversity and informational capacity in patients with DoC, along with reduced integration of the posterior default mode network with the rest of the brain [87"']. Unconsciousness was also associated with changes in the spontaneous hemodynamic response in the precuneus and posterior cingulate areas. This newly proposed measure allows investigators to study local metabolic changes and reflects the neurovascular coupling mechanism [88"].

fMRI can also provide insightful information when combined with passive stimulation, recently demonstrating that listening to the patient's favorite music generated increased connectivity compared to rest [89"]. Finally, fMRI-based active communication paradigms have not reached clinical implementation yet, but a simpler hand-raising motor task was able to detect appropriate brain activation in motor areas among DoC patients whose outcomes were more favorable than fMRI-unreactive patients [90].

MRI has advanced understanding of consciousness processes, through both structural quantitative methods and functional characterization of brain networks. However, its low accessibility, repeatability and its sensitivity to motion artifacts are major hurdles, and it has faced difficulties to translate mechanistic insights into clinically relevant information. The EAN guidelines on neuroimaging recommend the addition of fMRI sequences only in cases where a structural MRI is already indicated, and they highlight sedation and movement as confounding factors. Nevertheless, the guidelines acknowledge that active fMRI paradigms can detect cognitive motor dissociation with a high specificity and a low sensitivity, the latter possibly increased when using salient or familiar stimuli [2"'].

\section{FUNCTIONAL NEAR-INFRARED SPECTROSCOPY}

Functional near-infrared spectroscopy records the cerebral hemodynamic response in real-time and provides a safe, portable and noninvasive alternative to the logistical constraints of fMRI. It has been proposed as a feasible modality to develop braincomputer interfaces for noncommunicative patients. A recent study has achieved relatively accurate communication with healthy controls instructed to imagine playing tennis for 'yes' and stay relaxed for 'no', 
by decoding their neural response with support vector machine classifiers [91]. Additionally, concurrent analysis of resting-state near-infrared spectroscopy and clinical EEG allows to measure neurovascular coupling. Using this approach in the intensive care unit, unsupervised learning models were able to distinguish between patients in different states of consciousness and successfully identify those who later failed to recover [92"]. Functional near-infrared spectroscopy is thus a promising and practical neuroimaging modality that may reveal future groundbreaking applications for patients with DoC, but rigorous assessment of its reliability and further development of its clinical applications are necessary.

\section{MULTIMODAL STUDIES}

An increasing number of experimental protocols are using multimodal measures to investigate brain function in DoC. As each imaging modality suffers from inherent limitations, the parallel assessment of DoC patients with different devices increases the diagnostic accuracy and the likeliness to detect covert consciousness [93"']. These multimodal studies also shed light on the intricate relationship between independent neuroimaging biomarkers, as illustrated by the recent finding that EEG power spectra correlate with subcortical atrophy measured by structural MRI [94"]. Recent studies have also employed combined modalities to pinpoint the neural correlates of a specific behavior. Differences in brain activity between patients with visual pursuit and those with only a reflexive visual blink were demonstrated concurrently by EEG (N2/P2 components), MRI (integrity of optic radiations and primary visual cortex) and FDG-PET (calcarine cortex and lingual gyrus metabolism) [95"]. Similarly, unresponsive patients able to localize auditory stimuli were found to have increased fMRI connectivity between frontoparietal and visual areas, higher alpha-band EEG connectivity, and higher levels of brain metabolism than those without auditory localization [ $\left.7^{\mathbf{}}\right]$. In another study, the presence of increasingly complex auditory behaviors in patients with DoC correlated with graded preservation of brain function in language-related areas, assessed by auditory evoked potentials, FDG-PET, structural and functional MRI measures [96"].

\section{CONCLUSION}

Neuroimaging techniques have become fundamental components of the assessment of a brain-injured patient. Each modality possesses its strengths and limitations, and we advocate the development of multimodal protocols in which complementary data are used to increase diagnostic accuracy, develop prognostic models and identify the neural basis for conscious behaviors [97"']. To translate experimental results into actionable tools for clinicians, we highlight the need to implement recent guidelines and develop decision-making protocols based on sequential or parallel multimodal tests (Fig. 1) [98"]. We encourage the use of neuroimaging to better characterize brain processes at the acute and chronic stages after injury, which will lead to the elaboration of personalized therapies. Developing these treatments and assessing their efficacy will benefit from neuroimaging biomarkers, which may reveal evidence of therapeutic responses before behavioral effects are observed [40]. Finally, neuroimaging will play a key role in the systematic detection of patients with a capacity for covert consciousness, for which tailored streamlined care plans will need to be implemented in partnership with caregivers and families [99].

\section{Acknowledgements}

In the scope of the studies that were conducted by the Coma Science Group in Belgium, we would like to thank the patients, their families, and the staff from the departments of radiology, nuclear medicine and intensive care, at the University Hospital of Liège, Belgium.

\section{Financial support and sponsorship}

The study was supported by: the University and University Hospital of Liege, the Belgian National Funds for Scientific Research (FRS-FNRS), the European Union's Horizon 2020 Framework Programme for Research and Innovation under the Specific Grant Agreement No. 945539 (Human Brain Project SGA3), the European Space Agency (ESA) and the Belgian Federal Science Policy Office (BELSPO) in the framework of the PRODEX Programme, the Center-TBI project (FP7-HEALTH602150), the National Institutes of Health (NIH) Director's Office (DP2HD101400), the NIH National Institute of Neurological Disorders and Stroke (R21NS109627, RF1NS115268), the Public Utility Foundation 'Université Européenne du Travail', 'Fondazione Europea di Ricerca Biomedica', the BIAL Foundation, AstraZeneca Foundation, the Mind Science Foundation, the fund Generet, the King Baudouin Foundation, the Mind Care International foundation, the James S. McDonnell Foundation, the Tiny Blue Dot Foundation, and DOCMA project [EU-H2020-MSCARISE-778234]. L.R.D.S. is research fellow, O.G. is research associate, and S.L. is research director at the FRS-FNRS.

\section{Conflicts of interest}

There are no conflicts of interest. 


\section{REFERENCES AND RECOMMENDED} READING

Papers of particular interest, published within the annual period of review, have been highlighted as:

- of special interest

-1. of outstanding interest

1. Giacino JT, Katz DI, Schiff ND, et al. Practice guideline update recommendations summary: disorders of consciousness. Neurology 2018; 91:450-460.

2. Kondziella D, Bender $A$, Diserens $K$, et al. European Academy of Neurology

n. guideline on the diagnosis of coma and other disorders of consciousness. Eur J Neurol 2020; 27:741-756.

This landmark paper provides recommendations on the diagnosis of DoC by a guideline taskforce of 10 international experts appointed by the European Academy of Neurology. Twenty statements are issued following a systematic review of the literature and correspond to research questions elaborated with a Patient, Outcome, Comparator, Outcome (PICO) design, related to behavioral, neurophysiological and neuroimaging assessments.

3. Comanducci A, Boly M, Claassen J, et al. Clinical and advanced neurophy- siology in the prognostic and diagnostic evaluation of disorders of consciousness: review of an IFCN-endorsed expert group. Clin Neurophysiol $2020 ; 131: 2736-2765$

This narrative review endorsed by the International Federation of Clinical Neurophysiology examines the evidence for the use of neurophysiology in the diagnostic and prognostic evaluation of DoC. The panel of expert authors provides an overview of the available evidence on conventional clinical EEG, evoked potentials, quantitative EEG, transcranial magnetic stimulation, as well as active EEG paradigms, and recommendation statements are given for each technique.

4. Fins JJ. Disorders of consciousness, past, present, and future. Cambridge $\mathrm{Q}$ Healthc Ethics 2019; 28:603-615.

5. Rissman L, Paquette ET. Ethical and legal considerations related to disorders of consciousness. Curr Opin Pediatr 2020; 32:765-771.

6. Zasler ND, Aloisi M, Contrada M, Formisano R. Disorders of consciousness

- terminology: history, evolution and future directions. Brain Inj 2019; 33:1684-1689.

This review examines the evolution of the diagnostic terminology used to categorize DoC. It also sets a frame for the future development and standardization of this nomenclature, encouraging international consensus and highlighting the implications at stake.

7. Carrière $M$, Cassol $H$, Aubinet $C$, et al. Auditory localization should be

- considered as a sign of minimally conscious state based on multimodal findings. Brain Commun 2020; 2:fcaa195.

This article advocates the addition of auditory localization as a new criterion of the minimally conscious state, showing that unresponsive patients able to localize sounds have better neuroimaging profiles and clinical outcomes than those who do not demonstrate this clinical sign.

8. Hermann B, Salah A.B., Perlbarg V., et al. Habituation of auditory startle

- reflex is a new sign of minimally conscious state. Brain 2020; 143:2154-2172.

This article proposes the habituation of auditory startle reflex as a new clinical sign of the minimally conscious state. The authors investigated the cortically-mediated nature of this behavior and its ability to predict clinical outcomes at 6 months among patients with DoC.

9. Arzi A, Rozenkrantz L, Gorodisky L, et al. Olfactory sniffing signals consciousness in unresponsive patients with brain injuries. Nature 2020; $581: 428-433$.

10. Laureys S, Celesia GG, Cohadon F, et al. Unresponsive wakefulness syndrome: a new name for the vegetative state or apallic syndrome. BMC Med 2010; 8:2-5.

11. Van Erp WS, Lavrijsen JCM, Vos PE, et al. Unresponsive wakefulness syndrome: outcomes from a vicious circle. Ann Neurol 2020; 87:12-18.

12. Olaya J, Noé E, Navarro MD, et al. When, how, and to what extent are individuals with unresponsive wakefulness syndrome able to progress? Functional independence. Brain Sci 2020; 10:990.

13. Giacino JT, Ashwal $\mathrm{S}$, Childs $\mathrm{N}$, et al. The minimally conscious state. Neurology 2002; 58:349-353.

14. Thibaut A, Bodien YG, Laureys S, Giacino JT. Minimally conscious state 'plus': diagnostic criteria and relation to functional recovery. J Neurol 2020; 267:1245-1254

15. Bruno L M-A. Vanhaudenhuyse A, Thibaut $A$, et al. From unresponsive wakefulness to minimally conscious PLUS and functional locked-in syndromes: recent advances in our understanding of disorders of consciousness. J Neurol 2011; 258:1373-1384.

16. Bodien YG, Martens G, Ostrow J, et al. Cognitive impairment, clinical symptoms and functional disability in patients emerging from the minimally conscious state. NeuroRehabilitation 2020; 46:65-74.

17. Sherer M, Katz DI, Bodien YG, et al. Posttraumatic confusional state: a case definition and diagnostic criteria. Arch Phys Med Rehabil 2020; 101:2041-2050.

18. Giacino JT, Kalmar K, Whyte J. The JFK Coma Recovery Scale-Revised: measurement characteristics and diagnostic utility. Arch Phys Med Rehabil 2004; 85:2020-2029.
19. Wang J, Hu X, Hu Z, et al. The misdiagnosis of prolonged disorders of consciousness by a clinical consensus compared with repeated comarecovery scale-revised assessment. BMC Neurol 2020; 20:343.

20. Aubinet C, Cassol H, Bodart O, et al. Simplified Evaluation of CONsciousness Disorders (SECONDs) in individuals with severe brain injury: a validation study. Ann Phys Rehabil Med 2020; S1877-0657:30160-30163.

21. Pincherle A, Rossi F, Jöhr J, et al. Early discrimination of cognitive motor dissociation from disorders of consciousness: pitfalls and clues. J Neurol 2021; 268:178-188.

22. Annen J, Filippini MM, Bonin E, et al. Diagnostic accuracy of the CRS-R index in patients with disorders of consciousness. Brain Inj 2019; 33:1409-1412.

23. Pincherle $A$, Jöhr J, Chatelle $C$, et al. Motor behavior unmasks residual cognition in disorders of consciousness. Ann Neurol 2019; 85:443-447.

24. Bernard C, Delmas V, Duflos $C$, et al. Assessing pain in critically ill braininjured patients: a psychometric comparison of 3 pain scales and videopupillometry. Pain 2019; 160:2535-2543.

25. Sanz LRD, Aubinet $C$, Cassol $H$, et al. SECONDs administration guidelines: a fast tool to assess consciousness in brain-injured patients. J Vis Exp 2021; 168:e61968.

26. Schiff ND. Cognitive motor dissociation following severe brain injuries. JAMA Neurol 2015; 72:1413-1415.

27. Edlow BL, Claassen J, Schiff ND, Greer DM. Recovery from disorders of consciousness: mechanisms, prognosis and emerging therapies. Nat Rev Neurol 2020; 17:135-156.

28. Gosseries O, Zasler ND, Laureys S. Recent advances in disorders of consciousness: focus on the diagnosis. Brain Inj 2014; 28:1141-1150.

29. Monti MM, Vanhaudenhuyse $A$, Coleman MR, et al. Willful modulation of brain activity in disorders of consciousness. N Engl J Med 2010; 362:579-589.

30. Bruno MA, Soddu A, Demertzi A, et al. Disorders of consciousness: moving from passive to resting state and active paradigms. Cogn Neurosci 2010; $1: 193-203$.

31. Fischer D, Threlkeld ZD, Bodien $Y G$, et al. Intact brain network function in an unresponsive patient with COVID -19. Ann Neurol 2020; 88:851-854.

32. Boly M, Massimini M, Tsuchiya $N$, et al. Are the neural correlates of consciousness in the front or in the back of the cerebral cortex? Clinical and neuroimaging evidence. J Neurosci 2017; 37:9603-9613.

33. Ihalainen R, Gosseries $\mathrm{O}$, Van de Steen $\mathrm{F}$, et al. How hot is the hot zone? Computational modelling clarifies the role of parietal and frontoparietal connectivity during anaesthetic-induced loss of consciousness. Neuroimage 2021; 231:117841.

34. Bareham CA, Roberts N, Allanson J, et al. Bedside EEG predicts longitudinal

- behavioural changes in disorders of consciousness. Neurolmage Clin 2020; 28:102372.

This study used longitudinal high-density EEG recordings in prolonged DoC patients over 2 years and demonstrated that EEG measures were able to predict future changes in CRS-R scores and outperform clinical prognostic power.

35. Velly L, Perlbarg V, Boulier T, et al. Use of brain diffusion tensor imaging for the prediction of long-term neurological outcomes in patients after cardiac arrest: a multicentre, international, prospective, observational, cohort study. Lancet Neurol 2018; 17:317-326.

36. Claassen J, Doyle K, Matory A, et al. Detection of brain activation in unresponI. sive patients with acute brain injury. N Engl J Med 2019; 380:2497-2505. This study conducted in the intensive care unit employed machine learning on EEG recordings to detect brain activation in response to spoken commands, among clinically unresponsive patients. The authors reported that $15 \%$ of patients were able to modulate their brain activity to command in the first week after brain injury, and these patients had improved functional outcomes.

37. Sokoliuk R, Degano G, Banellis L, etal. Covert speech comprehension predicts - recovery from acute unresponsive states. Ann Neurol 2021; 89:646-656. This article demonstrated the prognostic value of a passive paradigm recording the EEG response to auditory streams of monosyllabic words among unresponsive patients with acute traumatic brain injury.

38. Estraneo A, Fiorenza S, Magliacano A, et al. Multicenter prospective study on predictors of short-term outcome in disorders of consciousness. Neurology 2020; 95:e1488-e1499.

39. Mensen A, Bodart O, Thibaut $A$, et al. Decreased evoked slow-activity after - tDCS in disorders of consciousness. Front Syst Neurosci 2020; 14:14:62. This study employed an innovative design to monitor the effects of treatment by transcranial direct current stimulation among patients with DoC. Using transcranial magnetic stimulation combined with EEG, it showed decreased slow-wave activity after stimulation compared to before the intervention.

40. Thibaut A, Schiff N, Giacino J, et al. Therapeutic interventions in patients with prolonged disorders of consciousness. Lancet Neurol 2019; 18:600-614.

41. Alkhachroum $A$, Eliseyev $A$, Der-Nigoghossian CA, et al. EEG to detect early

- recovery of consciousness in amantadine-treated acute brain injury patients. J Neurol Neurosurg Psychiatry 2020; 91:675-676.

This study used EEG to monitor the effects of amantadine treatment on patients with prolonged DoC before clinical effects could be observed with standardized behavioral scales.

42. Edlow BL, Barra ME, Zhou DW, et al. Personalized connectome mapping to guide targeted therapy and promote recovery of consciousness in the intensive care unit. Neurocrit Care 2020; 33:364-375. 
43. Bonin EAC, Lejeune N, Thibaut $A$, et al. Nociception coma scale-revised allows to identify patients with preserved neural basis for pain experience. J Pain 2020; 21:742-750.

44. Kane N. Disorders of consciousness: a role for clinical neurophysiology? Clin Neurophysiol 2020; 131:2732-2733.

45. Bai $Y$, Lin Y, Ziemann U. Managing disorders of consciousness: the role of - electroencephalography. J Neurol 2020; In Press.

This systematic review provides a comprehensive overview of the EEG measurements currently available to assist the diagnosis, prognosis and therapy monitoring of DoC, and it evaluates their relative performance, either individually or in multivariate models.

46. Engemann DA, Raimondo F, King J-R, et al. Robust EEG-based cross-site and cross-protocol classification of states of consciousness. Brain 2018; 141:3179-3192.

47. Annen J, Mertel I, Xu R, et al. Auditory and somatosensory P3 are com-

- plementary for the assessment of patients with disorders of consciousness. Brain Sci 2020; 10:748.

This study demonstrates that ERPs induced by auditory and vibrotactile stimulations have independent performance to detect consciousness, which encourages the use of multimodal ERP protocols.

48. Boly M, Garrido MI, Gosseries O, et al. Preserved feedforward but impaired top-down processes in the vegetative state. Science 2011; 332:858-862.

49. Wu M, Li F, Wu Y, et al. Impaired frontoparietal connectivity in traumatic individuals with disorders of consciousness: a dynamic brain network analysis. Aging Dis 2020; 11:301-314.

50. Møller MLH, Højlund A, Jensen M, et al. Applied potential of task-free event-

- related paradigms for assessing neurocognitive functions in disorders of consciousness. Brain Commun 2020; 2:fcaa087.

This focused review examines the available evidence on the different paradigms using ERPs for the diagnosis of DoC, and proposes a new multidimensional approach combining well studied ERP components in a single paradigm to improve diagnostic accuracy.

51. Laforge G, Gonzalez-Lara LE, Owen AM, Stojanoski B. Individualized as-

- sessment of residual cognition in patients with disorders of consciousness. Neurolmage Clin 2020; 28:102472.

This paper reports the use of a passive movie-watching task to detect covert consciousness, which provides a naturalistic tool to assess language-processing abilities.

52. Curley $\mathrm{WH}$, Forgacs $\mathrm{PB}$, Voss $\mathrm{HU}$, et al. Characterization of EEG signals revealing covert cognition in the injured brain. Brain 2018; 141:1404-1421.

53. Goldfine AM, Victor JD, Conte MM, et al. Determination of awareness in patients with severe brain injury using EEG power spectral analysis. Clin Neurophysiol 2011; 122:2157-2168.

54. Cruse D, Chennu $S$, Chatelle $C$, et al. Bedside detection of awareness in the vegetative state: a cohort study. Lancet 2011 ; 378:2088-2094.

55. Gui $P$, Jiang $Y$, Zang $D$, et al. Assessing the depth of language processing in patients with disorders of consciousness. Nat Neurosci 2020; 23:761-770.

56. Braiman $\mathrm{C}$, Fridman $\mathrm{EA}$, Conte $\mathrm{MM}$, et al. Cortical response to the natural speech envelope correlates with neuroimaging evidence of cognition in severe brain injury. Curr Biol 2018; 28:3833-3839. e3.

57. Chatelle C, Rosenthal ES, Bodien YG, et al. EEG correlates of language

- function in traumatic disorders of consciousness. Neurocrit Care 2020; 33:449-457

This study compares the performance of four different EEG-based methods to assess residual language functions in traumatic DoC patients. The results showed that visual EEG evaluation methods with standardized criteria and automated classifiers were both useful but in different clinical settings.

58. Gibson RM, Ray LB, Laforge G, et al. 24-h polysomnographic recordings and electrophysiological spectral analyses from a cohort of patients with chronic disorders of consciousness. J Neurol 2020; 267:3650-3663.

59. Forgacs PB, Conte MM, Fridman EA, et al. Preservation of electroencephalographic organization in patients with impaired consciousness and imagingbased evidence of command-following. Ann Neurol 2014; 76:869-879.

60. Mertel I, Pavlov YG, Barner C, et al. Sleep in disorders of consciousness:

- behavioral and polysomnographic recording. BMC Med 2020; 18:350.

This epidemiological study reported that patients with DoC demonstrated a distribution of EEG sleep features significantly different from healthy controls and conscious tetraplegic patients, which suggests that the disruption of sleep architecture observed in DoC is independent from immobilization and hospital environment.

61. Casarotto S, Comanducci A, Rosanova M, et al. Stratification of unresponsive patients by an independently validated index of brain complexity. Ann Neurol 2016; 80:718-729.

62. Sinitsyn DO, Poydasheva AG, Bakulin IS, et al. Detecting the potential for

- consciousness in unresponsive patients using the perturbational complexity index. Brain Sci 2020; 10:917.

This recent study successfully replicated, on an independent dataset, the diagnostic performance of the perturbational complexity index. This measure derived from transcranial magnetic stimulation combined with EEG allows the accurate discrimination between unconscious and conscious patients based on a single metric.

63. Lutkenhoff ES, Johnson MA, Casarotto S, et al. Subcortical atrophy correlates with the perturbational complexity index in patients with disorders of consciousness. Brain Stimul 2020; 13:1426-1435.
64. Pauli R, O'Donnell A, Cruse D. Resting-state electroencephalography for - prognosis in disorders of consciousness following traumatic brain injury. Front Neurol 2020; 11:586945.

This review focuses on the prognostic methods using resting-state EEG to predict the outcomes of patients after traumatic brain injury, and concludes that the lack of long-term data calls for further research on the performance of very long-term EEG prognostic tools.

65. Varrone A, Asenbaum S, Vander Borght T, et al. EANM procedure guidelines for PET brain imaging using [18F]FDG, version 2. Eur J Nucl Med Mol Imaging 2009; 36:2103-2110.

66. Thibaut A., Panda R., Annen J., et al. Preservation of brain activity in unresponsive patients identifies MCS star. Ann Neurol 2021; In Press.

67. Stender J, Gosseries O, Bruno M-A, et al. Diagnostic precision of PET imaging and functional MRI in disorders of consciousness: a clinical validation study. Lancet 2014; 384:514-522.

68. Kondziella D, Friberg CK, Frokjaer VG, et al. Preserved consciousness in vegetative and minimal conscious states: systematic review and meta-analysis. J Neurol Neurosurg Psychiatry 2016; 87:485-492.

69. Candia-Rivera D, Annen J, Gosseries $O$, et al. Neural responses to heartbeats detect residual signs of consciousness during resting state in postcomatose patients. J Neurosci 2021; JN-RM-1740-20.

70. Stender J, Mortensen KN, Thibaut $A$, et al. The minimal energetic requirement of sustained awareness after brain injury. Curr Biol 2016; 26:1494-1499.

71. Aubinet $\mathrm{C}$, Cassol $\mathrm{H}$, Gosseries $\mathrm{O}$, et al. Brain metabolism but not gray

- matter volume underlies the presence of language function in the minimally conscious state (MCS): MCS + Versus MCS - Neuroimaging Differences. Neurorehabil Neural Repair 2020; 34:172-184.

This multimodal study investigated the differences in neuroimaging features between MCS - and MCS + patients, and found that FDG-PET brain metabolism in language-related areas was significantly higher in MCS + patients, despite similar grey matter volumes assessed by MRI.

72. Edlow BL, Sanz LRD, Polizzotto L, et al. the Curing Coma Campaign and its contributing members: therapies to restore consciousness in patients with severe brain injuries: a gap analysis and future directions. Neurocrit Care 2021; In Press.

73. Snider SB, Edlow BL. MRI in disorders of consciousness. Curr Opin Neurol

I. 2020; 33:676-683.

This comprehensive review article discusses the recent advances in structural and functional MRI applied to the study of DoC. It highlights the novel applications of resting-state functional connectivity and the paradigms allowing to detect covert consciousness.

74. Golkowski D, Larroque SK, Vanhaudenhuyse A, et al. Changes in whole brain dynamics and connectivity patterns during sevoflurane- and propofol-induced unconsciousness identified by functional magnetic resonance imaging. Anesthesiology 2019; 130:898-911.

75. Demertzi A, Tagliazucchi E, Dehaene $S$, et al. Human consciousness is supported by dynamic complex patterns of brain signal coordination. Sci Adv 2019; 5:eaat7603.

76. Li X, Tan X, Wang P, et al. Chronic disorders of consciousness: a case report with longitudinal evaluation of disease progression using $7 \mathrm{~T}$ magnetic resonance imaging. BMC Neurol 2020; 20:396.

77. Stafford CA, Owen AM, Fernández-Espejo D. The neural basis of external responsiveness in prolonged disorders of consciousness. Neurolmage Clin 2019; $22: 101791$

78. Snider SB, Bodien YG, Frau-Pascual A, et al. Ascending arousal network

- connectivity during recovery from traumatic coma. Neurolmage Clin 2020; 28:102503.

This study uses longitudinal diffusion tensor imaging measures to investigate structural connectivity changes in the ascending arousal network of patients after acute traumatic coma. The results demonstrate an increased connectivity between the brainstem and the thalamus as well as other significant differences among the group who recovered.

79. Snider SB, Bodien YG, Bianciardi M, et al. Disruption of the ascending arousal network in acute traumatic disorders of consciousness. Neurology 2019; 93:e1281-e1287.

80. Fernández-Espejo $D$, Soddu $A$, Cruse $D$, et al. A role for the default mode network in the bases of disorders of consciousness. Ann Neurol 2012; $72: 335-343$

81. Rudas J, Martínez D, Castellanos G, et al. Time-delay latency of resting-state blood oxygen level-dependent signal related to the level of consciousness in patients with severe consciousness impairment. Brain Connect 2020; 10:83-94.

82. Martínez DE, Rudas J, Demertzi $A$, et al. Reconfiguration of large-scale functional connectivity in patients with disorders of consciousness. Brain Behav 2020; 10:e1476.

83. Varley TF, Craig M, Adapa R, et al. Fractal dimension of cortical functional connectivity networks \& severity of disorders of consciousness. PLoS One 2020; 15:e0223812.

84. Crone JS, Lutkenhoff ES, Vespa PM, Monti MM. A systematic investigation of the association between network dynamics in the human brain and the state of consciousness. Neurosci Conscious 2020; 2020:niaa008.

85. Cao $\mathrm{B}, \mathrm{Chen} \mathrm{Y}, \mathrm{Yu} \mathrm{R}$, et al. Abnormal dynamic properties of functional connectivity in disorders of consciousness. Neurolmage Clin 2019; 24:102071.

86. Threlkeld ZD, Bodien YG, Rosenthal ES, et al. Functional networks reemerge during recovery of consciousness after acute severe traumatic brain injury. Cortex 2018; 106:299-308. 
87. Luppi Al, Craig MM, Pappas I, et al. Consciousness-specific dynamic interac-1. tions of brain integration and functional diversity. Nat Commun 2019; 10:4616. This study combines resting-state functional MRI data from healthy controls, anesthetized subjects and DoC patients, to identify consciousness-specific patterns of dynamic functional connectivity. The authors demonstrate that unconscious states are associated with specific characteristics of functional networks in terms of integration, segregation and functional diversity.

88. Wu G-R, Di Perri C, Charland-Verville V, et al. Modulation of the spontaneous

- hemodynamic response function across levels of consciousness. Neuroimage 2019; 200:450-459.

This study uses the spontaneous hemodynamic response, a measure based on resting-state $\mathrm{FMRI}$, to characterize the neurovascular coupling process in the brain of propofol-anesthetized subjects and UWS patients.

89. Carrière M, Larroque SK, Martial C, et al. An echo of consciousness: brain - function during preferred music. Brain Connect 2020; 10:385-395.

This article reports resting-state fMRI functional connectivity increases among patients with DoC listening to their favorite music, compared to the rest condition, and these effects were dependent on the level of consciousness. There results encourage further assessment of music therapy for the recovery of DoC patients.

90. Wang F, Hu N, Hu X, et al. Detecting brain activity following a verbal command in patients with disorders of consciousness. Front Neurosci 2019; 13:976.

91. Abdalmalak A, Milej D, Yip LCM, et al. Assessing time-resolved fNIRS for brain-computer interface applications of mental communication. Front Neurosci 2020; 14:105.

92. Othman $\mathrm{MH}$, Bhattacharya $\mathrm{M}$, Møller $\mathrm{K}$, et al. Resting-State NIRS-EEG in

- unresponsive patients with acute brain injury: a proof-of-concept study. Neurocrit Care 2020; 34:31-44.

This proof-of-concept study investigated the use of functional near-infrared spectroscopy combined with EEG to assess neurovascular coupling among unresponsive patients in the intensive care unit. This measure revealed to be sensitive to changing consciousness levels and could identify patients who failed to recover consciousness with a high accuracy.

93. Schnakers $\mathrm{C}$, Hirsch M, Noé E, et al. Covert cognition in disorders of m. consciousness: a meta-analysis. Brain Sci 2020; 10:930.

This important meta-analysis reviewed the available evidence on paradigms designed to detect covert cognition and investigated whether specific clinical or demographic factors contributed to the chance of responding to an active neuroimaging paradigm. Data from 25 studies were eligible for analysis and revealed that traumatic etiologies are more likely to respond to an active task, whereas clinical diagnosis (UWS or MCS-) does not influence the likelihood to respond.
94. Lutkenhoff ES, Nigri A, Rossi Sebastiano D, et al. EEG Power spectra and - subcortical pathology in chronic disorders of consciousness. Psychol Med 2020; 1-10; In Press.

This cross-sectional multimodal study studied for the first time the correlations between EEG spectral measures and MRI-based grey matter subcortical atrophy among patients with prolonged DoC

95. Sattin D, Rossi Sebastiano D, D'Incerti L, et al. Visual behaviors in disorders

- of consciousness: disentangling conscious visual processing by a multimodal approach. Eur J Neurosci 2020; 52:4345-4355.

This multimodal study investigated the integrity of the visual system using EEG, MRI and FDG-PET among patients manifesting a visual pursuit compared to those showing only a reflexive visual blink.

96. Ferraro S, Nigri A, D'Incerti L, et al. Preservation of language processing and

- auditory performance in patients with disorders of consciousness: a multimodal assessment. Front Neurol 2020; 11:526465.

This article describes the results of a multimodal assessment of auditory functions among patients with prolonged DoC. EEG, MRI and FDG-PET measures were compared to the scores obtained in the auditory subscale of the CRS-R and provided neuroimaging correlates for auditory behaviors.

97. Jain R, Ramakrishnan AG. Electrophysiological and neuroimaging studies -

-1 during resting state and sensory stimulation in disorders of consciousness: a review. Front Neurosci 2020; 14:555093.

This extensive review provides a wide overview on the use of neurophysiological and neuroimaging techniques in the assessment of patients with DoC. The authors compare different modalities, stimulation and resting-state paradigms, emotional content of stimuli or analytic methods, and give insights into future directions of research and clinical practice.

98. Schnakers C, Monti MM. Towards improving care for disorders of conscious- ness. Nat Rev Neurol 2020; 16:405-406.

This comment reflects on the recent publication of guidelines by both the American and the European Academies of Neurology on DoC, and highlights the milestones they represent. The authors call for their implementation and the elaboration of a scientific agenda in future research on DoC, which would encompass the use of multimodal neuroimaging tools and the development of new therapeutic options.

99. Jöhr J, Halimi F, Pasquier J, et al. Recovery in cognitive motor dissociation after severe brain injury: a cohort study. PLoS One 2020; 15:e0228474.

100. Bodart O, Gosseries $O$, Wannez $S$, et al. Measures of metabolism and complexity in the brain of patients with disorders of consciousness. NeuroImage Clin 2017; 14:354-362. 